\title{
Special issue: Multi-agent systems for medicine, computational biology, and bioinformatics
}

Recent progresses in the area of biomedical sciences, including medicine, bioinformatics and computational biology has made it essential to devise new insights and technological platforms able to ensure appropriate support to research activities. To facilitate the most relevant tasks, a new generation of systems, tools, and applications needs to be developed, aimed at ensuring the feasibility of significant advances in all relevant subfields of biomedicine.

Medical and biological information is increasing at an impressive rate, and it is still typically made available to researchers through web interfaces - which are actually mainly suitable to human beings rather than to software applications. Doctors and biologists that work on the border line of state-of-the-art research topics are accustomed to connect to many sites characterized by heterogeneous interfaces and data structures, as well as to switch among them in order to submit and to retrieve preliminary and intermediate data. It is clear that no generic description can be given, able to encompass all requirements imposed by different disciplines and kinds of researchers, nevertheless -in all cases- researchers must deal with complex systems and envision a kind of "entropic change", with the feeling that a new era for systems, tools, and applications is approaching. Note that "complex", here, does not necessarily address computational problems. Rather, the complexity lies in the conceptualization, analysis, and design of the system, tool, or application to be implemented, as well as in the interactions among entities (despite their "nature" of objects, components, or agents) that are involved at run-time.

Several cross-sectional issues occur in the cited biomedical sciences. For the sake of brevity, let us consider only: (i) the problem of integrating heteroge- neous information and resources, (ii) the problem of simulating and modelling complex biological systems, and (iii) the problem of providing relevant specific tools and applications, possibly via web interfaces. As for the integration of heterogeneous information and resources, any solution should be given keeping in mind that an essential requirement is the openness of the proposal - to ensure that newly structured information can be processed, provided that a suitable ontological description of it is given. As for the simulation and modeling of complex biological systems (see [Bosse et al.], [Bandini et al.], [Merelli and Young]), which are crucial issues in systems biology and computational biology, it is clear the need for specific tools and notations. The need for distribution (in order to speed-up the corresponding computation), and for the adoption of software paradigms able to deal with concurrencyrelated issues are further relevant concerns. As for the need of specific tools and applications, usually supplied through web interfaces and finalized to problemsolving, visualization, or other analytical tasks (see [Bortolussi and Dovier]), it is clear that they must undergo a rapid change in order to support new technologies, e.g. web services, and to provide results according to a holistic view (see [Koutkias et al.]).

Although the path forward is still long and difficult, the agent technology is now being assessed in biomedicine, also for its ability of dealing with information integration, distribution, and with concurrencyrelated issues. It is worth pointing out that the paradigmatic change issued by the adoption of this new technology would not imply a dramatic change, as agents can encompass existing applications, through suitable wrappers, as well as new technologies, such as web services, through suitable gateways. 
As a final remark, I would like to thank all authors for their active commitment and their willing to make this special issue successful. In fact, this special issue is the result of a remarkable effort made by the authors, which revised their paper in order to produce a high-quality product, so that the interested reader can be gently introduced in the world envisioned by the authors - and shared by myself - in which software agents can be used to solve various biomedical problems, ranging from the handling of medical information to the simulation of complex systems.

The guest-editor

Giuliano Armano

Dept. of Electrical and Electronical Engineering

University of Cagliari

Italy

E-mail:armano@diee.unica.it 